\title{
Geometric compatibility of IceCube TeV-PeV neutrino excess and its galactic dark matter origin
}

\author{
Yang Bai, ${ }^{a}$ Ran $\mathbf{L u}^{a, b}$ and Jordi Salvado ${ }^{a, c}$ \\ ${ }^{a}$ Department of Physics, University of Wisconsin, \\ University Avenue, Madison, WI 53706, U.S.A. \\ ${ }^{b}$ Michigan Center for Theoretical Physics, University of Michigan, \\ Church Street, Ann Arbor, MI 48109, U.S.A. \\ ${ }^{c}$ Wisconsin IceCube Particle Astrophysics Center, \\ West Washington Avenue, Madison, WI 53706, U.S.A. \\ E-mail: yangbai@physics.wisc.edu, rlu@wisc.edu, \\ jordi.salvado@ific.uv.es
}

AbSTRACT: We perform a geometric analysis for the sky map of the IceCube TeV-PeV neutrino excess and test its compatibility with the sky map of decaying dark matter signals in our galaxy. We have found that a galactic decaying dark matter component in general improve the goodness of the fit of our model, although the pure isotropic hypothesis has a better fit than the pure dark matter one. We also consider several representative decaying dark matter, which can provide a good fit to the observed spectrum at IceCube with a dark matter lifetime of around 12 orders of magnitude longer than the age of the universe.

KeYwords: Neutrino Detectors and Telescopes

ARXIV EPRINT: 1311.5864 


\section{Contents}

1 Introduction

2 Geometric analysis for decaying dark matter based on the KolmogorovSmirnov test

3 Geometric analysis using the likelihood ratio test

6

4 Neutrino spectra from dark matter decays

5 Conclusions and discussion

\section{Introduction}

One of the important tasks for physicists is to understand the nature of dark matter (DM). The indirect search of DM from its self-annihilation or decay serves as a promising approach to learn additional interactions of DM with Standard Model (SM) particles. Among many potential products from DM annihilation or decay, neutrino serves a useful candidate because its propagation is less disturbed by the interstellar medium and its observation may point out the DM geometric distribution in the galaxy.

The existing searches of DM from cosmic neutrinos have been concentrated on the galactic center, dwarf galaxies, clusters of galaxies or the center of the Sun [1-4]. All previous searches have found good agreements between the neutrino spectrum and the predicted astrophysical background. The story has been changed from the observation of 53 high energy neutrino events at IceCube for neutrino energy above around $30 \mathrm{TeV}$ [5-7], which is well above the predicted number of the background events $[8,9]^{1}$ and has a $7.0 \sigma$ inconsistence with the standard atmospheric backgrounds.

This IceCube result is based on data taken between May 2010 and May 2014 using detectors with 79 and 86 strings and has a total integrated time of approximately 1347 days. The observed 53 events have two events slightly above $1 \mathrm{PeV}$ [13] and one around $2 \mathrm{PeV}[6]$, the remaining 50 events with an energy between $25 \mathrm{TeV}$ and $0.4 \mathrm{PeV}$. The observed events can also be divided into "track" and "cascade" events, depending on event shapes. The track events are most likely produced by muon neutrinos via charge-current interactions, while the cascade events could come from electron neutrinos with chargecurrent interactions or all types of neutrinos with neutral-current interactions. Among the 53 events, the 14 track events have a good angular resolution with around $1^{\circ}$ uncertainty

\footnotetext{
${ }^{1}$ Recent discussion about the prompt atmospheric neutrino background from charms and background uncertainties from the parton distribution functions can be found in refs. [10, 11] and ref. [12], respectively.
} 
around the event direction, while the other 39 cascade events have poor angular resolutions ranging from $\sim 10^{\circ}$ to $\sim 50^{\circ}$.

The angular resolutions of those events play an important role for identifying the geometric origin of the neutrino excess. The IceCube collaboration has performed a point source analysis for the 4 years cascade events and found that there is no significant evidence of spatial clustering and the $p$-value for the hypothesis of a uniform event distribution is $18 \%$ [7]. Curious about the possible linkage between the TeV-PeV neutrino excess at IceCube and the mysterious DM in our universe, in this paper we analyze the IceCube data with a special attention on its geometric distributions, and study the statistical significance of its potential DM origin, which prefers to have more signal events around the galactic center because of the DM spatial profile.

One could consider DM annihilation as an explanation. However, due to the unitarity bound $[14,15]$, we found that the annihilation rate for a DM mass around one $\mathrm{PeV}$ is about four orders of magnitude lower than the required one for the IceCube data. Therefore, we concentrate on the decaying DM case, which can match to the required rate for a DM lifetime of $10^{28}-10^{29} \mathrm{~s}$. In this paper we do not provide a theoretical understanding of the DM mass scale and the decay lifetime, but we want to point out that a heavy DM with a non-thermal history has been widely predicted in many models [16-19].

Decaying dark matter as a potential source for these neutrino events (focusing on the energy spectrum) has been considered in the literature [19-22]. Here we present a geometric analysis which is complimentary to the previous studies. Before entering into the details, we would like to point out other recent studies for the IceCube neutrino excess including cosmogenic productions via photo-meson interactions [11, 23-27], galactic sources [26-30], active galactic nuclei [25, 31, 32], gamma-ray bursts [25, 33, 34], starforming galaxies [35, 36], and a leptoquark beyond the SM [37].

In our following statistical analysis, we will perform both the Kolmogorov-Smirnov test and the likelihood ratio test using the official release of the IceCube data for the four years [7]. The KS test will answer how compatible of different model hypotheses with the experimental data. The likelihood test will concentrate on comparing different model hypotheses directly. Those two tests are complimentary to each other: the KS test could exclude all model hypothesis using the features in experimental data; the likelihood test may tell us the individual weights of a combination of models.

\section{Geometric analysis for decaying dark matter based on the Kolmogorov- Smirnov test}

Our main goal is to study the compatibility of the neutrino sky map from DM and the sky map of the observed events at IceCube. The signal distribution from DM decays depends on the DM spacial profile in our galaxy. For the Einasto profile [38], one has

$$
\rho_{\mathrm{DM}}(r)=\rho_{\odot} e^{-\frac{2}{\bar{\alpha}}\left[\left(\frac{r}{r_{s}}\right)^{\bar{\alpha}}-\left(\frac{r_{\odot}}{r_{s}}\right)^{\bar{\alpha}}\right]},
$$

with $r_{s}=20 \mathrm{kpc}$ and $\bar{\alpha}=0.17$ as a standard choice. A larger value of $\bar{\alpha}$ has a flatter DM profile. Here, $r_{\odot} \simeq 8.5 \mathrm{kpc}$ is the distance from the Sun to the galactic center; $\rho_{\odot} \simeq$ 

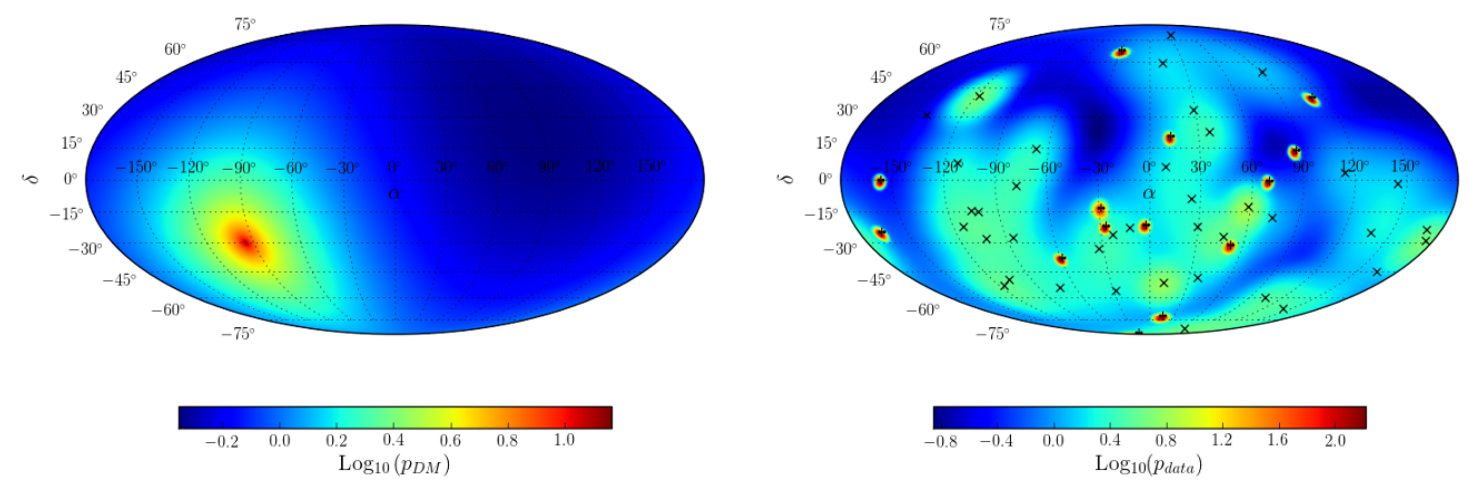

Figure 1. Left panel: the sky map of the neutrinos from decaying DM with an Einasto profile in eq. (2.1). Right panel: the sky map of the IceCube 53 events after taking into account the angular resolution. The seven red spots correspond to the seven "track" events.

$0.3 \mathrm{GeV} \mathrm{cm}^{-3}$ is the approximate DM density in the solar system. The neutrino signal from DM decay is calculated by the line-of-sight integral along a given direction [18]

$$
\frac{d \Phi_{\nu}}{d E_{\nu} d b d l}=\frac{d N}{N d E_{\nu}} \frac{1}{\tau_{\mathrm{DM}} m_{\mathrm{DM}}} \frac{\cos b}{4 \pi} \int d s \rho_{\mathrm{DM}}[r(s)]
$$

where the integral of $s$ is along the line of sight and the relation between $r$ and $s$ is $r^{2}=s^{2}+r_{\odot}^{2}-2 s r_{\odot} \cos l \cos b$, where $-90^{\circ} \leq b<90^{\circ}$ and $-180^{\circ} \leq l<180^{\circ}$ as the latitude and longitude angles in the galactic coordinate. $\tau_{\mathrm{DM}}$ is the DM lifetime and $m_{\mathrm{DM}}$ is the DM mass. The normalized neutrino differential spectrum is $d N /\left(N d E_{\nu}\right)$. The integrated neutrino flux from DM is

$$
\Phi_{\nu}=1.7 \times 10^{-12} \mathrm{~cm}^{-2} \mathrm{~s}^{-1} \mathrm{sr}^{-1} \times \frac{10^{28} s}{\tau_{\mathrm{DM}}} \times \frac{1 \mathrm{PeV}}{m_{\mathrm{DM}}} .
$$

For the integrated time of 1347 days and $10 \mathrm{~m}^{2} \cdot \mathrm{sr}$ acceptance area for the energy around $100 \mathrm{TeV}$, there could be around 20 events observed at IceCube.

The geometric distribution of the IceCube events is represented in the equatorial coordinate. We, therefore, translate the DM generated event distribution from the galactic coordinate in the latitude and longitude angles $(b, l)$ to the equatorial coordinate in the declination angle and the right ascension angle $(\delta, \alpha)$ (see ref. [39] for details). We define the DM probability distribution using the normalized flux

$$
p_{\mathrm{DM}}(\delta, \alpha)=\frac{1}{\Phi_{\nu}} \frac{d \Phi_{\nu}(\delta, \alpha)}{d \delta d \alpha},
$$

with the DM event sky map shown in the left panel of figure 1. For all or subsets of the observed 53 events from IceCube, we construct the data probability distribution using the solid-angular error $\sigma_{i}$ for each event by assuming a Gaussian distribution

$$
p_{\text {data }}^{N \text { events }}(\delta, \alpha)=\frac{1}{N} \sum_{i \in N} \frac{1}{2 \pi \sigma_{i}^{2}} \exp \left[-\frac{\Delta R\left(\delta_{i}, \alpha_{i} ; \delta, \alpha\right)^{2}}{2 \pi \sigma_{i}^{2}}\right],
$$


where $\Delta R\left(\delta_{i}, \alpha_{i} ; \delta, \alpha\right)$ is the angular distance between the points $\left(\delta_{i}, \alpha_{i}\right)$ and $(\delta, \alpha)$ on the sphere. In the right panel of figure 1 , we show the sky map of the observed $N=53$ events at IceCube after implementing the angular resolution for each event. Comparing these two maps, one can see that both have a concentration of events around the galactic center direction. On the other hand, the DM sky map has very few events in the right and upper corner, while the IceCube data map has some population in this region.

To quantify the similarity of the two sky maps in figure 1, we perform a statistical test to calculate the $p$-value of the hypothesis of decaying DM as an explanation of IceCube neutrino excess. We first use a two-dimensional version of the Kolmogorov-Smirnov (KS) test statistics (TS) [40] to study the compatibility between the data and the DM hypothesis. We will use the maximum likelihood-ratio test as well later. The KS test statistics is defined as the largest absolute difference between cumulative probability distributions of the data and the model. It takes better account of the relation among data points than the traditional likelihood-ratio test.

To make the definition of the TS less sensitive to the integration directions, we consider a set of four possible integration regions,

$$
\begin{aligned}
S\left(\delta_{0}, \alpha_{0}\right)=\{ & \left(\delta<\delta_{0}, \alpha<\alpha_{0}\right),\left(\delta>\delta_{0}, \alpha<\alpha_{0}\right), \\
& \left.\left(\delta<\delta_{0}, \alpha>\alpha_{0}\right),\left(\delta>\delta_{0}, \alpha>\alpha_{0}\right)\right\},
\end{aligned}
$$

for a given boundary choice $\left(\delta_{0}, \alpha_{0}\right)$. The TS or the difference of the cumulative probability distributions is defined by

$$
\operatorname{TS}\left(\delta_{0}, \alpha_{0}\right) \equiv \sup _{r \in S\left(\delta_{0}, \alpha_{0}\right)}\left|\int_{r} d \delta d \alpha p_{\text {model }}(\delta, \alpha)-\int_{r} d \delta d \alpha p_{\text {data }}(\delta, \alpha)\right| .
$$

Choosing the largest value for all possible boundary choices, we have the KS test statistics as

$$
\mathrm{TS}_{\mathrm{KS}}=\sup \left\{\bigcup_{\operatorname{all}\left(\delta_{0}, \alpha_{0}\right)} \operatorname{TS}\left(\delta_{0}, \alpha_{0}\right)\right\} .
$$

To calculate the $p$-value for the decaying DM as an explanation for the data, we generate random event maps by choosing a random (according to the model profile) rightascension angle but keeping the same declination angle and resolution of the event in the data. Notice that scrambling the events only in the right-ascencion does not introduce any bias in the analysis, having one of the coordinates fixed, it only incorporates an equal shift in the $\chi^{2}$ for every random realization and the data. If the data is not in a very unlikely realization the effect of using only right-ascencion in the p-value calculation in general it reduce the power for the test statistic moreover we chose to do that to avoid possible bias introduced by the systematic effects such that earth absorption in the zenith distribution. This same technique is used for the point sources analysis done by the IceCube collaboration $[5,6]$ with the same data.

In the left panel of figure 2, we show the TS distribution of the reference decaying DM model against maps of randomly sampled 53 events. The red vertical line indicates the 

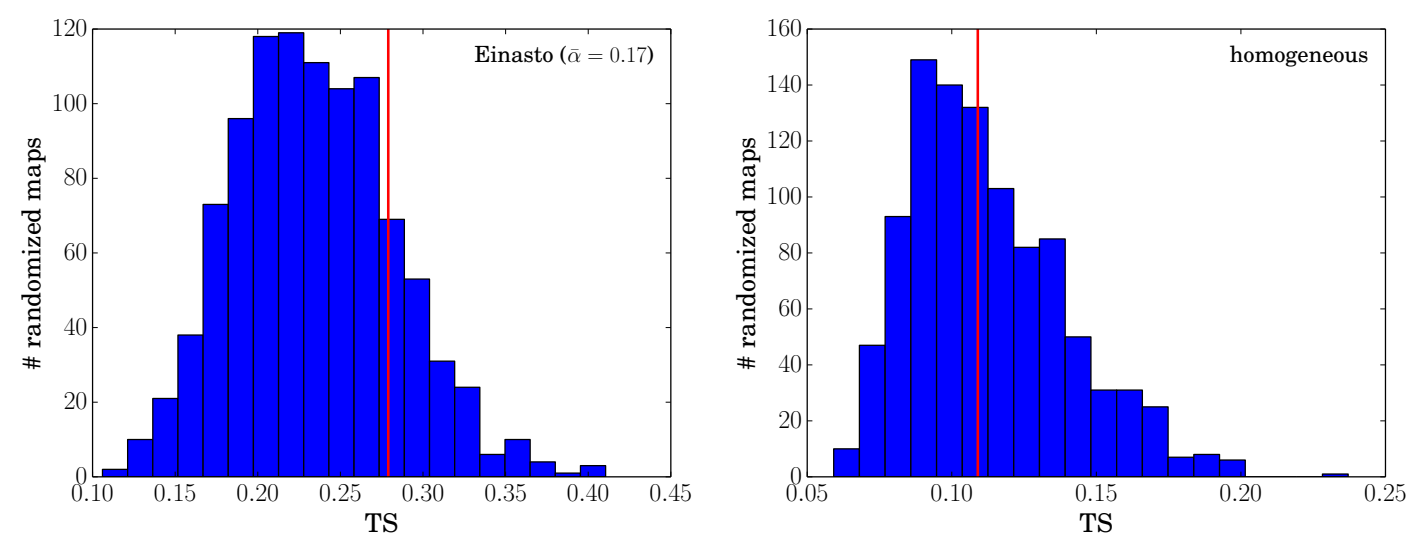

Figure 2. Left panel: the TS distribution for the Einasto model with $\bar{\alpha}=0.17\left(p_{\text {value }}=18.9 \%\right)$ against random sky maps with random right-ascension angles for the 53 events. Right panel: the TS distribution for a isotropic distribution $\left(p_{\text {value }}=46.4 \%\right)$. The TS value for the data is shown on the red lines.

\begin{tabular}{|c|c|c|c|}
\hline & $\bar{\alpha}=0.17$ & $\bar{\alpha}=0.25$ & Isotropic \\
\hline all 53 events & $18.9 \%$ & $15.2 \%$ & $46.4 \%$ \\
\hline 34 events with $E \gtrsim 50 \mathrm{TeV}$ & $11 \%$ & $17.8 \%$ & $69.4 \%$ \\
\hline 39 cascade events & $34.1 \%$ & $28.9 \%$ & $74.2 \%$ \\
\hline
\end{tabular}

Table 1. The $p$-value's for three different hypothesis's using all the events, only the events with $E \gtrsim 50 \mathrm{TeV}$ and only the cascade events.

test statistics TS(DM) of DM against the observed 53 events at IceCube. The $p$-value, or the probability of having $\mathrm{TS}(\mathrm{DM})$ smaller than the TS value from a random event map, is $36.8 \%$ for the Einasto model with $\bar{\alpha}=0.17$. To test how good the observed 53 events agree with a isotropic geometrical distribution, we perform the same calculation by assuming a isotropic model (in the right panel of figure 2) and found that the $p$-value for a isotropic distribution is $49.8 \%$ for all 53 events.

Since the atmospheric backgrounds are dominated in lower energies [8, 9], a bigger fraction of the observed events could be from DM signals if only relatively high energy events are selected. Therefore, we also test the geometric distributions for the 34 events with $E \gtrsim 50 \mathrm{TeV}$. We show the $p$-values for all 53 events and the 34 events with $E \gtrsim 50 \mathrm{TeV}$ in table 1 . One can see that the $p$-values are fairly insensitive to the energy cut. In the last row of table 1 , we also show the $p$-values for only the cascade events considering the fact that the track events could have an origin from the atmospheric muon background. From table 1, one can already see that there is no dramatic difference between $\bar{\alpha}=0.25$ and $\bar{\alpha}=0.17$ cases. This is due to the poor angular resolution of cascade events such that the peaked center of the DM profiles can not be resolved. The increase of the $p$-values for the isotropic distribution from all 53 events to 39 cascade events is due to the extremely good resolution of the 14 track events. 


\section{Geometric analysis using the likelihood ratio test}

In the last section we saw that all the hypothesis are statistically compatible with the different sets of data. The next question to ask would be how much of the signal events may come from the different hypothesis and how can we exclude only Isotropic or only DM against a combination of the two. In this section we check the compatibility of the data with the DM profile using a likelihood ratio test, which was used by the IceCube collaboration in their point source analysis [41]. We first treat the isotropic distribution as the null hypothesis with an alternative isotropic plus DM hypothesis. We define the likelihood function as

$$
\mathcal{L}\left(n_{s}\right)=\prod_{i}^{N}\left[\frac{n_{s}}{N} \mathcal{S}_{i}+\left(1-\frac{n_{s}}{N}\right) \mathcal{B}_{i}\right],
$$

where $\mathcal{B}_{i}$ is the isotropic background contribution and $\mathcal{S}_{i}$ is the signal DM contribution. ${ }^{2}$ The $n_{s}$ is the number of signal events and will be used to maximize the likelihood. We use the observed data locations and errors convoluted by the DM probability distribution to calculate the signal contribution $\mathcal{S}_{i}$ as

$$
\mathcal{S}_{i}=\int \frac{1}{2 \pi \sigma_{i}^{2}} e^{-\frac{\left|\vec{x}_{i}-\vec{x}_{s}\right|}{2 \sigma_{i}^{2}}} p_{\mathrm{DM}}\left(\vec{x}_{s}\right) d^{2} \vec{x}_{s} .
$$

Here, $\vec{x}_{i}$ is a vector, defined in the $(\delta, \alpha)$ plane, from the location of the observed event and $\sigma_{i}$ is the corresponding angular error. We show the log-likelihood function as a function of signal strength $n_{s} / N$ in figure 3 for three cases: all 53 events, 34 events with $E \gtrsim 50 \mathrm{TeV}$ and 39 cascade events. We can see from figure 3 that the preferred values of $n_{s}$ are positive, which suggests that a combination of DM plus isotropic distributions fit the data better than the isotropic-only fit. Comparing the best fitted values of $n_{s}$ for $\bar{\alpha}=0.17$ and $\bar{\alpha}=0.25$, one can see that a larger value of $\bar{\alpha}$ or a flatter DM profile prefers more DM signal events.

To quantify the $p$-value of the data to reject the isotropic-only hypothesis against the isotropic plus DM hypothesis, we calculate the test statistic as

$$
\mathrm{TS}=\max _{n_{s}}\left\{2 \log \left[\frac{\mathcal{L}\left(n_{s}\right)}{\mathcal{L}(0)}\right]\right\} .
$$

As we did in the last section we compute the $p$-values by scrambling the events in right ascension angle $\alpha$ with a distribution consistent with the background. For all the 53 IceCube events, we show the histogram for the TS distribution in figure 4 and have the vertical and red line at the real data location. For all three choices of events, we show the $p$-values and the $n_{s}$ 's at the maximum likelihood for two different values of $\bar{\alpha}$ in table 2 , which clearly show that the 39 cascade events have a smaller value for the isotropic-only hypothesis.

All the cases are statistically consistent with the null hypothesis, therefore additional galactic component coming from the DM decay in our galaxy is not needed to explain

\footnotetext{
${ }^{2}$ We have also calculated the $p$-values for the point source signal hypothesis and found a good agreement with the result obtained by the IceCube collaboration [5].
} 


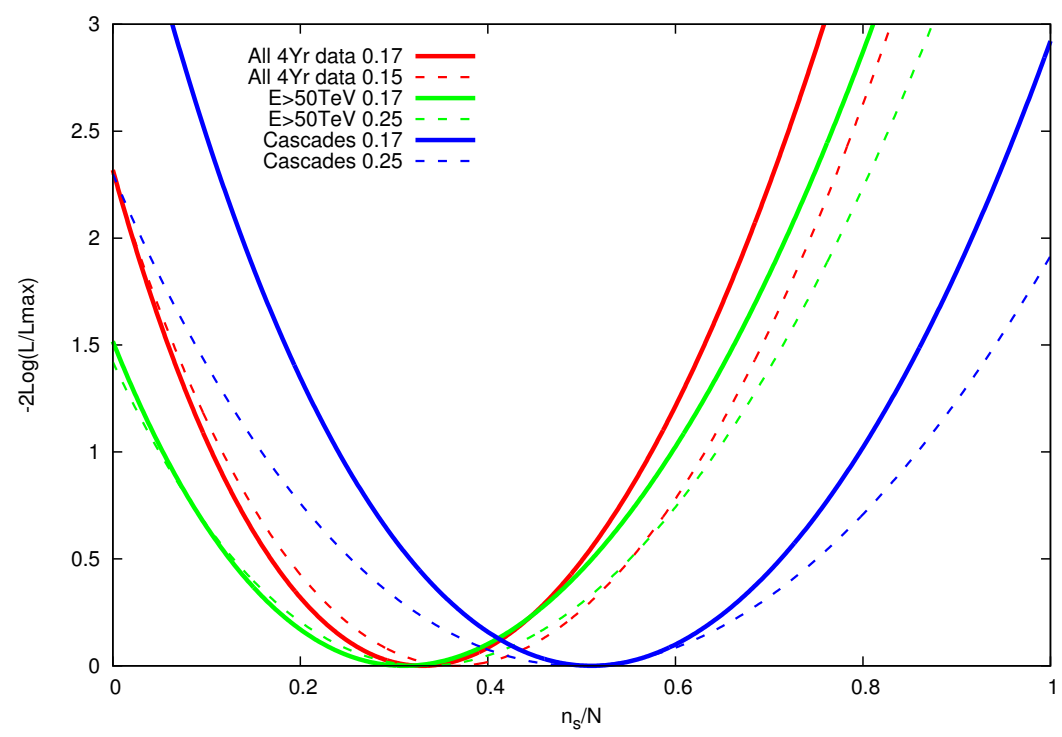

Figure 3. The log-likelihood as a function of the signal event fraction for all 53 events, 34 events with $E \gtrsim 50 \mathrm{TeV}$ and 39 cascade events. The solid lines are for $\bar{\alpha}=0.17$ and the dotted lines are for $\bar{\alpha}=0.25$.

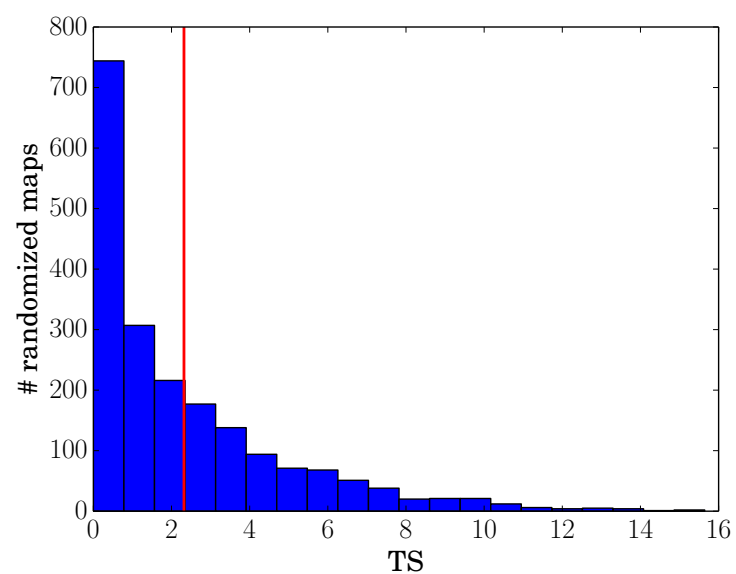

Figure 4. The TS distribution for the Einasto model with $\bar{\alpha}=0.17\left(p_{\text {value }}=36.8 \%\right)$, the red line is the TS for all 53 events. Here, we have $\mathcal{S}=\mathrm{DM}$ and $\mathcal{B}=$ isotropic, to have the isotropic distribution as the null hypothesis.

\begin{tabular}{|c|c|c|}
\hline & $\bar{\alpha}=0.17$ & $\bar{\alpha}=0.25$ \\
\hline all 53 events & $36.8 \%(17)$ & $38.0 \%(19)$ \\
\hline 34 events with $E \gtrsim 50 \mathrm{TeV}$ & $43.18 \%(10)$ & $46.8 \%(11)$ \\
\hline 39 cascade events & $18.1 \%(19)$ & $10.06 \%(21)$ \\
\hline
\end{tabular}

Table 2. The $p$-values using the likelihood method using all the events, only the events with $E \gtrsim 50 \mathrm{TeV}$ and only the cascade events. The numbers in the parenthesis are the numbers of signal events after maximize the log-likelihood. Here, we have $\mathcal{S}=\mathrm{DM}$ and $\mathcal{B}=$ isotropic in eq. (3.1). 
the current data. On the other hand the data is still not enough to exclude the DM hypothesis. In this case we would expect some galactic and extragalactic DM fluxes that may be comparable [42]. This estimation is still consistent with the best fit number of signal events that we see in the current data, table 2. In the future the IceCube [43] extension may bring enough statistics to potentially exclude the DM origin of the majority neutrino events.

\section{Neutrino spectra from dark matter decays}

The energy spectrum of the IceCube neutrino excess has interesting features [5]. First, there are three events with deposited energy above $1 \mathrm{PeV}$ [6]. Secondly, there is an potential energy cutoff below the Glashow resonance energy. Although a wide range of the energy spectrum can be fit by an $E^{-2}$ feature [5], it is still interesting to explore potential DM produced spectra from particle physics.

To fit the observed spectrum at IceCube, one also needs to consider different detector acceptances at different energies. For different flavors of neutrinos, the acceptance areas vary a lot with the largest one for the electron neutrino. In our analysis below, we don't distinguish different flavors of neutrinos and use the averaged acceptance areas in terms of flavors and declination angles [5], which for the flavor part is equivalent to assume the 1:1:1 combination. This effective area is only slightly different from ref. [24].

Because the uncertainties on the acceptance areas and the large statistical errors, the current IceCube data is not sufficient to distinguish spectra among different particle physics models. So, we consider several representative decaying DM models and study their fit to the observed energy spectrum. We consider candidate models according to the operator dimensions of DM coupling to SM particles.

At the renormalizable level and for a fermion DM $\chi$, we consider the operator $\lambda H \bar{L}_{L} \chi$ for DM coupling to the Higgs field in the SM or $\lambda H_{L} \bar{L}_{L} \chi$ in the lepton-specific two-Higgs doublet models, which has DM decays as $\chi \rightarrow h+l$ and $\chi \rightarrow l+H_{L} \rightarrow l+\tau^{+}+\tau^{-}$, respectively. Setting the fermion DM mass to $8 \mathrm{PeV}$, it is possible to fit the spectra observed by IceCube. For a scalar DM, one can have the renormalizable coupling to the SM Higgs boson as simple as $\mu X \mathrm{HH}^{\dagger}$, which simply mediates the decay of $X \rightarrow 2 h$. Because the neutrino spectrum is relatively soft, the mass of $\mathrm{DM}$ has to be raised to $20 \mathrm{TeV}$ for a reasonable fit. Beyond the renormalizable level, one could have DM mainly couple to two leptons via $\epsilon m_{\tau} X \tau^{+} \tau^{-} / \Lambda$, so the decay channel is $X \rightarrow \tau^{+} \tau^{-}$. Such DM with $8 \mathrm{TeV}$ mass can fit the IceCube spectrum well. We show the fitted spectra in figure 5 after using PYTHIA [44] for SM particles decay and hadronization. (see [19, 20] for other spectra from DM decays).

\section{Conclusions and discussion}

Our geometrical analysis has already shown that a combination of the galactic DM contribution and an isotropic spectrum provides the best fit to the data. Because there are neutrino events pointing to the direction where the DM density is expected to be quite 


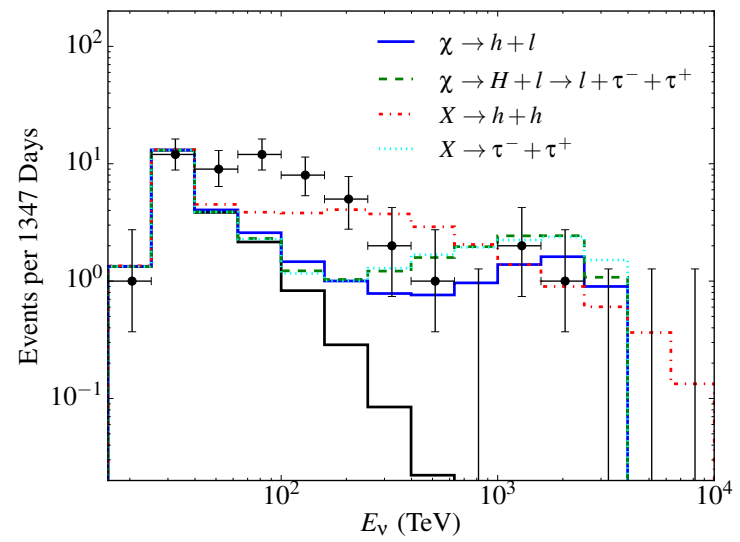

Figure 5. The fitted spectra for several DM decay channels. The black and solid line is the atmospheric backgrounds [8,9]. For the two fermion DM cases, the DM mass is $8 \mathrm{PeV}$ and both lifetimes are $\tau_{\chi}=2.3 \times 10^{28} \mathrm{~s}$. For the scalar DM decays into two higgses, the DM mass is $20 \mathrm{PeV}$ and the lifetimes are $4.6 \times 10^{28} \mathrm{~s}$. The DM decaying into $\tau^{-}+\tau^{+}$is $8 \mathrm{PeV}$, with lifetime $2.3 \times 10^{28} \mathrm{~s}$, respectively.

low, a purely galactic DM origin for the 53 events is excluded unless a flatter DM spacial profile like the isothermal one is used. It is also important to notice that extra-galactic DM may also produce an extra homogeneous contribution to the signal. A more detailed analysis of the DM distributions in the universe is needed to address this possibility.

If the significance of the source at the center of our galaxy keeps growing as the data accumulates, decaying dark matter will remain a strong candidate for at least part of the TeV-PeV neutrino events observed by the IceCube experiment. The IceCube has more data to be analyzed and collected, so a more robust conclusion can be drawn in the coming years. Other than IceCube, another neutrino telescope, ANTARES [45], has reached a comparable sensitivity in some declination angle region. A geometric test for the compatibility between the neutrinos (excess) observed in ANTARES and a decaying DM will be interesting.

Beyond the neutrino signal from DM, one could also search for other correlated and for sure model-dependent cosmic ray signatures from the DM decays at other experiments like Fermi LAT [46, 47], PAMELA [48, 49], AMS-02 [50] and HESS [50]. In the few respective models considered, additional photons, positrons and antiprotons can be produced at the same time when a neutrino signal is generated. One can easily check that the neutrino yield is considerably higher than the photon, positron and antiproton yields in every bin. Furthermore, because of the long DM lifetime of $10^{28}-10^{29} \mathrm{~s}$, the predicted photon, positron and antiproton fluxes have been checked to satisfy the current cosmic ray constraints (see ref. [35] for the gamma ray prediction at Fermi-LAT for the hadronuclear origin of the IceCube neutrino excess).

The PeV scale DM considered here is definitely beyond the scope of high energy collider searches. If additional interactions exist between DM and quarks, the direct detection experiments may see a signature [51]. If the IceCube excess is indeed due to decaying DM, a new avenue to understanding the DM properties will be opened. 


\section{Acknowledgments}

We would like to thank Vernon Barger, Daniel Chung, Francis Halzen, Claudio Kopper, Naoko Neilson and Nathan Whitehorn for useful discussion. The work is supported by the U.S. Department of Energy under the contract DE-FG-02-95ER40896. YB is partially supported by startup funds from the UW-Madison. The work of RL is partially supported by U.S. Department of Energy under the contract DE-FG-02-95ER40899 and by the Michigan Center for Theoretical Physics. JS acknowledges support from the Wisconsin IceCube Particle Astrophysics Center.

Open Access. This article is distributed under the terms of the Creative Commons Attribution License (CC-BY 4.0), which permits any use, distribution and reproduction in any medium, provided the original author(s) and source are credited.

\section{References}

[1] F. Halzen and D. Hooper, The Indirect Search for Dark Matter with IceCube, New J. Phys. 11 (2009) 105019 [arXiv:0910.4513] [InSPIRE].

[2] Super-Kamiokande collaboration, T. Tanaka et al., An Indirect Search for WIMPs in the Sun using 3109.6 days of upward-going muons in Super-Kamiokande, Astrophys. J. 742 (2011) 78 [arXiv: 1108.3384] [INSPIRE].

[3] IcECuBE collaboration, M.G. Aartsen et al., Search for dark matter annihilations in the Sun with the 79-string IceCube detector, Phys. Rev. Lett. 110 (2013) 131302 [arXiv:1212.4097] [INSPIRE].

[4] ANTARES collaboration, S. Adrian-Martinez et al., First results on dark matter annihilation in the Sun using the ANTARES neutrino telescope, JCAP 11 (2013) 032 [arXiv:1302.6516] [INSPIRE].

[5] IcECube collaboration, M.G. Aartsen et al., Evidence for High-Energy Extraterrestrial Neutrinos at the IceCube Detector, Science 342 (2013) 1242856 [arXiv:1311.5238] [INSPIRE].

[6] IceCube collaboration, M.G. Aartsen et al., Observation of High-Energy Astrophysical Neutrinos in Three Years of IceCube Data, Phys. Rev. Lett. 113 (2014) 101101 [arXiv: 1405.5303] [INSPIRE].

[7] ICECube collaboration, M.G. Aartsen et al., The IceCube Neutrino Observatory Contributions to ICRC 2015 Part II: Atmospheric and Astrophysical Diffuse Neutrino Searches of All Flavors, arXiv:1510.05223 [INSPIRE].

[8] M. Honda, T. Kajita, K. Kasahara, S. Midorikawa and T. Sanuki, Calculation of atmospheric neutrino flux using the interaction model calibrated with atmospheric muon data, Phys. Rev. D 75 (2007) 043006 [astro-ph/0611418] [inSPIRE].

[9] R. Enberg, M.H. Reno and I. Sarcevic, Prompt neutrino fluxes from atmospheric charm, Phys. Rev. D 78 (2008) 043005 [arXiv: 0806. 0418] [inSPIRE].

[10] P. Lipari, Establishing the astrophysical origin of a signal in a neutrino telescope, arXiv: 1308.2086 [INSPIRE]. 
[11] R. Laha, J.F. Beacom, B. Dasgupta, S. Horiuchi and K. Murase, Demystifying the PeV Cascades in IceCube: Less (Energy) is More (Events), Phys. Rev. D 88 (2013) 043009 [arXiv: 1306.2309] [INSPIRE].

[12] C.-Y. Chen, P.S. Bhupal Dev and A. Soni, Standard model explanation of the ultrahigh energy neutrino events at IceCube, Phys. Rev. D 89 (2014) 033012 [arXiv:1309.1764] [InSPIRE].

[13] IceCube collaboration, M.G. Aartsen et al., First observation of PeV-energy neutrinos with IceCube, Phys. Rev. Lett. 111 (2013) 021103 [arXiv: 1304.5356] [INSPIRE].

[14] K. Griest and M. Kamionkowski, Unitarity Limits on the Mass and Radius of Dark Matter Particles, Phys. Rev. Lett. 64 (1990) 615 [inSPIRE].

[15] L. Hui, Unitarity bounds and the cuspy halo problem, Phys. Rev. Lett. 86 (2001) 3467 [astro-ph/0102349] [INSPIRE].

[16] D.J.H. Chung, E.W. Kolb and A. Riotto, Superheavy dark matter, Phys. Rev. D 59 (1999) 023501 [hep-ph/9802238] [INSPIRE].

[17] D.J.H. Chung, E.W. Kolb and A. Riotto, Nonthermal supermassive dark matter, Phys. Rev. Lett. 81 (1998) 4048 [hep-ph/9805473] [INSPIRE].

[18] L. Covi, M. Grefe, A. Ibarra and D. Tran, Neutrino Signals from Dark Matter Decay, JCAP 04 (2010) 017 [arXiv: 0912.3521] [INSPIRE].

[19] B. Feldstein, A. Kusenko, S. Matsumoto and T.T. Yanagida, Neutrinos at IceCube from Heavy Decaying Dark Matter, Phys. Rev. D 88 (2013) 015004 [arXiv:1303.7320] [InSPIRE].

[20] A. Esmaili and P.D. Serpico, Are IceCube neutrinos unveiling PeV-scale decaying dark matter?, JCAP 11 (2013) 054 [arXiv:1308.1105] [INSPIRE].

[21] L.A. Anchordoqui et al., IceCube neutrinos, decaying dark matter and the Hubble constant, Phys. Rev. D 92 (2015) 061301 [arXiv: 1506.08788] [INSPIRE].

[22] C.S. Fong, H. Minakata, B. Panes and R.Z. Funchal, Possible Interpretations of IceCube High-Energy Neutrino Events, JHEP 02 (2015) 189 [arXiv:1411.5318] [INSPIRE].

[23] I. Cholis and D. Hooper, On The Origin of IceCube's PeV Neutrinos, JCAP 06 (2013) 030 [arXiv: 1211.1974] [INSPIRE].

[24] L.A. Anchordoqui, H. Goldberg, M.H. Lynch, A.V. Olinto, T.C. Paul and T.J. Weiler, Pinning down the cosmic ray source mechanism with new IceCube data, Phys. Rev. D 89 (2014) 083003 [arXiv:1306.5021] [INSPIRE].

[25] W. Winter, Photohadronic Origin of the TeV-PeV Neutrinos Observed in IceCube, Phys. Rev. D 88 (2013) 083007 [arXiv: 1307.2793] [INSPIRE].

[26] S. Razzaque, The Galactic Center Origin of a Subset of IceCube Neutrino Events, Phys. Rev. D 88 (2013) 081302 [arXiv:1309.2756] [INSPIRE].

[27] M. Ahlers and K. Murase, Probing the Galactic Origin of the IceCube Excess with Gamma-Rays, Phys. Rev. D 90 (2014) 023010 [arXiv:1309.4077] [INSPIRE].

[28] M.C. Gonzalez-Garcia, F. Halzen and V. Niro, Reevaluation of the Prospect of Observing Neutrinos from Galactic Sources in the Light of Recent Results in Gamma Ray and Neutrino Astronomy, Astropart. Phys. 57-58 (2014) 39 [arXiv:1310.7194] [INSPIRE].

[29] D.B. Fox, K. Kashiyama and P. Mészarós, Sub-PeV Neutrinos from TeV Unidentified Sources in the Galaxy, Astrophys. J. 774 (2013) 74 [arXiv:1305.6606] [InSPIRE]. 
[30] A. Neronov, D.V. Semikoz and C. Tchernin, PeV neutrinos from interactions of cosmic rays with the interstellar medium in the Galaxy, Phys. Rev. D 89 (2014) 103002 [arXiv: 1307.2158] [INSPIRE].

[31] O.E. Kalashev, A. Kusenko and W. Essey, PeV neutrinos from intergalactic interactions of cosmic rays emitted by active galactic nuclei, Phys. Rev. Lett. 111 (2013) 041103 [arXiv:1303.0300] [INSPIRE].

[32] F.W. Stecker, PeV neutrinos observed by IceCube from cores of active galactic nuclei, Phys. Rev. D 88 (2013) 047301 [arXiv: 1305.7404] [INSPIRE].

[33] K. Murase and K. Ioka, TeV-PeV Neutrinos from Low-Power Gamma-Ray Burst Jets inside Stars, Phys. Rev. Lett. 111 (2013) 121102 [arXiv:1306.2274] [INSPIRE].

[34] S. Razzaque, Long-lived PeV-EeV neutrinos from gamma-ray burst blastwave, Phys. Rev. D 88 (2013) 103003 [arXiv:1307.7596] [INSPIRE].

[35] K. Murase, M. Ahlers and B.C. Lacki, Testing the Hadronuclear Origin of PeV Neutrinos Observed with IceCube, Phys. Rev. D 88 (2013) 121301 [arXiv:1306.3417] [InSPIRE].

[36] R.-Y. Liu, X.-Y. Wang, S. Inoue, R. Crocker and F. Aharonian, Diffuse PeV neutrinos from EeV cosmic ray sources: Semirelativistic hypernova remnants in star-forming galaxies, Phys. Rev. D 89 (2014) 083004 [arXiv:1310.1263] [INSPIRE].

[37] V. Barger and W.-Y. Keung, Superheavy Particle Origin of IceCube PeV Neutrino Events, Phys. Lett. B 727 (2013) 190 [arXiv: 1305.6907] [InSPIRE].

[38] A.W. Graham, D. Merritt, B. Moore, J. Diemand and B. Terzic, Empirical Models for Dark Matter Halos. II. Inner profile slopes, dynamical profiles and $\rho / \sigma^{3}$, Astron. J. 132 (2006) 2701 [astro-ph/0608613] [INSPIRE].

[39] T. Neunhoffer, Estimating the angular resolution of tracks in neutrino telescopes based on a likelihood analysis, Astropart. Phys. 25 (2006) 220 [astro-ph/0403367] [INSPIRE].

[40] J.A. Peacock, Two-dimensional goodness-of-fit testing in astronomy, Mon. Not. Roy. Astron. Soc. 202 (1983) 615.

[41] J. Braun, J. Dumm, F. De Palma, C. Finley, A. Karle and T. Montaruli, Methods for point source analysis in high energy neutrino telescopes, Astropart. Phys. 29 (2008) 299 [arXiv:0801.1604] [INSPIRE].

[42] M. Ahlers, Y. Bai, V. Barger and R. Lu, Galactic TeV-PeV Neutrinos, arXiv:1505.03156 [INSPIRE].

[43] IceCube collaboration, M.G. Aartsen et al., IceCube-Gen2: A Vision for the Future of Neutrino Astronomy in Antarctica, arXiv:1412.5106 [INSPIRE].

[44] T. Sjöstrand, S. Mrenna and P.Z. Skands, A Brief Introduction to PYTHIA 8.1, Comput. Phys. Commun. 178 (2008) 852 [arXiv:0710.3820] [INSPIRE].

[45] ANTARES collaboration, S. Adrian-Martinez et al., Search for Cosmic Neutrino Point Sources with Four Year Data of the ANTARES Telescope, Astrophys. J. 760 (2012) 53 [arXiv: 1207.3105] [INSPIRE].

[46] Fermi-LAT collaboration, M. Ackermann et al., Constraints on the Galactic Halo Dark Matter from Fermi-LAT Diffuse Measurements, Astrophys. J. 761 (2012) 91 [arXiv: 1205.6474] [INSPIRE]. 
[47] Fermi-LAT collaboration, A.A. Abdo et al., Observations of Milky Way Dwarf Spheroidal galaxies with the Fermi-LAT detector and constraints on Dark Matter models, Astrophys. J. 712 (2010) 147 [arXiv: 1001.4531] [INSPIRE].

[48] PAMELA collaboration, O. Adriani et al., An anomalous positron abundance in cosmic rays with energies 1.5-100 GeV, Nature 458 (2009) 607 [arXiv:0810.4995] [INSPIRE].

[49] PAMELA collaboration, O. Adriani et al., PAMELA results on the cosmic-ray antiproton flux from $60 \mathrm{MeV}$ to $180 \mathrm{GeV}$ in kinetic energy, Phys. Rev. Lett. 105 (2010) 121101 [arXiv: 1007.0821] [INSPIRE].

[50] AMS collaboration, M. Aguilar et al., First Result from the Alpha Magnetic Spectrometer on the International Space Station: Precision Measurement of the Positron Fraction in Primary Cosmic Rays of 0.5-350 GeV, Phys. Rev. Lett. 110 (2013) 141102 [INSPIRE].

[51] I.F.M. Albuquerque and L. Baudis, Direct detection constraints on superheavy dark matter, Phys. Rev. Lett. 90 (2003) 221301 [Erratum ibid. 91 (2003) 229903] [astro-ph/0301188] [INSPIRE]. 